\title{
Applicability of Specific Trend's Consistency of Full Hydrogenation
}

\author{
Muhammad Yusuf Ritonga* \\ Department of Chemical Engineering, Faculty of Engineering, University of Sumatera Utara, \\ Medan 20155, Indonesia; yusufrit@gmail.com
}

\begin{abstract}
This paper reports the application of specific trend's consistency of Full Hydrogenation (FH) of SCPOFA to establish operating conditions for providing new product of Partially Hydrogenated (PH) fatty acid, an Intermediate Raw Material (IRM) of a mixed fatty acid T40, which is a modification of fatty acid products. Consistency of FH and prediction of fatty acid iodine value of SCPOFA produced the suitable quality of IRM for T40 by trial and error method. The initiating conditions to start PH of SCPOFA were constant; start temperature of $146^{\circ} \mathrm{C}$, pressure of $-1 \mathrm{bar}$, Ni catalyst dosage of $217 \mathrm{~kg} / \mathrm{ton}$ and agitation speed of $190 \mathrm{rpm}$. This reaction time produced the end temperature of $164^{\circ} \mathrm{C}$, and pressure of $4.6 \mathrm{bars}$. These results proved a simple practical method applicable under field conditions for industrial production of oleo chemical and edible oil.
\end{abstract}

Keywords: Specific Trend's Consistency, Full Hydrogenation, Partial Hydrogenation, T40 Quality, Operating Conditions

\section{Introduction}

Hydrogenation process has to be adopted to purify the Fatty Acids (FA) in oleo chemical industry, and Oils \& Fats (ONF) for edible oil industry. In case of diversification of FA and ONF products, the manipulation of operating conditions for hydrogenation (feed quality, temperature, pressure, kind and amount of metal catalyst and agitation speed) has to be done. The most practical industrial method has to be supported by theory, data, and practical field experiences (FA hydrogenation in oleo chemical industry, ONF hydrogenation in edible oil industry). How can this be done? This paper will discuss practical trend's specific consistency of full hydrogenation, applied to manipulate Hydrogenated Fatty Acid (HFA), and Hydrogenated Oil \& Fat (HONF) product qualities. A concept based on this method will be provided in this study for oleo chemical industry.

\subsection{Concepts and Significance of the Specific Trend's Consistency of Hydrogenation}

The simple mechanism of hydrogenation of fatty acids, oils and fats are shown as first chemical reaction (1) (Patterson) ${ }^{1}$. The simpler mechanisms are shown as second reaction $(2)^{2}$.

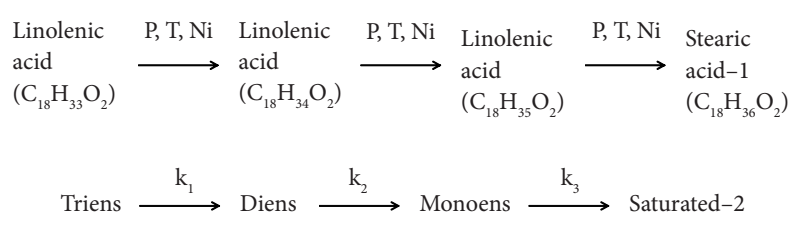

Operating conditions will limit hydrogenation reaction and the product quality. It can be hydrogenated partially or fully resulting in changing of physical and chemical properties of HFA, ONF, confirmed by

${ }^{*}$ Author for correspondence 
researchers ${ }^{3,4}$. Different conditions will give different trend's hydrogenation, but specificity and consistency is requ-ired for the specific operating conditions ${ }^{5}$. Effects of hydrogenation reaction time for some trends of physical properties of ONF given in Figure 1 established some consistent trend ${ }^{5}$. The experiences in the field confirmed this statement also, known as Specific Trend's Consistency of Full Hydrogenation.

Based on Figure 1, the reaction time can predict the changes of the direction of operating conditions (reaction temperature, pressure), physical properties (melting point, titer, solid fat index SFI, refractive index, and also reduction of chemical properties such as IV of FA oil and fats or vise versa. The specific quality of physi$\mathrm{cal}$, and chemical properties, which are on the range of the trend of specific properties can be predicted. All properties given in Figure 1 can be found in the range of reaction time and IV. The principle of determination is based on reaction time and IV, because time is needed to change the IV. The changing of IV itself is the indication of changing of FA, oil and fat properties. Reaction time is the common practice used in the monitoring of FA, and ONF hydrogenation. The questions to be addressed are: How to predict IV of HFA, and HNOF which will be made at a specific operating conditions, and quality? How can it be used into the specific trend consistency of hydrogenation?

Hydrogenation of FA, ONF, which contain linoleic and oleic acid begins from linoleic acid ${ }^{2}$. In full

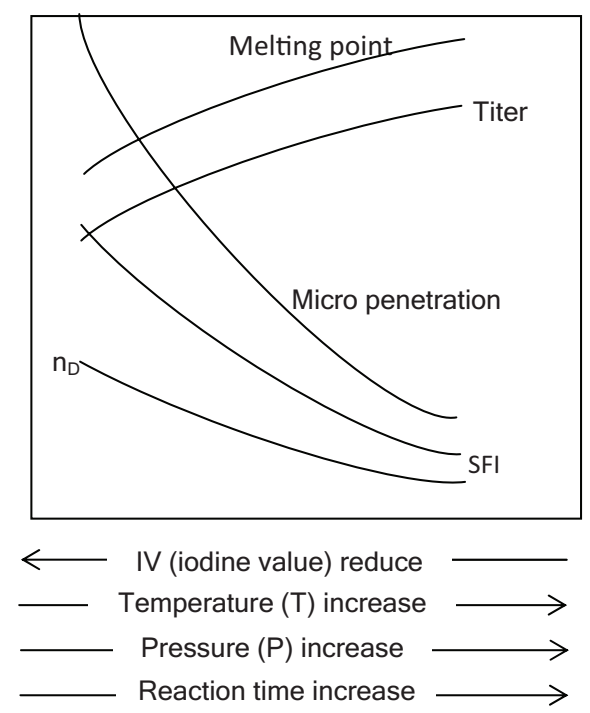

Figure 1. Reaction time affects to T, P, IV and the trends of some physical properties of ONF. hydrogenation of FA and ONF (as raw material), all unsaturated fatty acids change to become saturated FA, and ONF. Figure 2 and Figure 3 show the specific trend's consistency of full hydrogenation of Splitted CPO Fatty Acid (SCPOFA) on the same specific operating conditions. Based on these, the iodine value, composition of $\mathrm{C}_{18-2}, \mathrm{C}_{18-1}$ and $\mathrm{C}_{18}$ in hydrogenated SCPOFA, temperature and pressure of reaction in the reactor (operating conditions) can be recorded and evaluated at specific levels - on the specific reaction time of fatty acids hydrogenation - easily. It means that some specific qualities of HSCPOFA can be able to manipulate and produce the intermediate raw materials for the next purification or products, and also the final products in oleo chemical industry. These are the manipulation of operating conditions and product qualities.

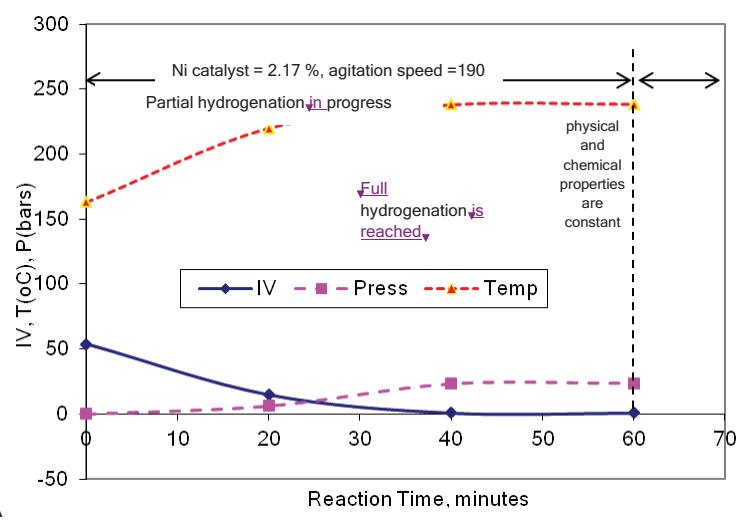

Figure 2. The specific trend's consistency of IV, T and P for full hydrogenation of SCPOFA.

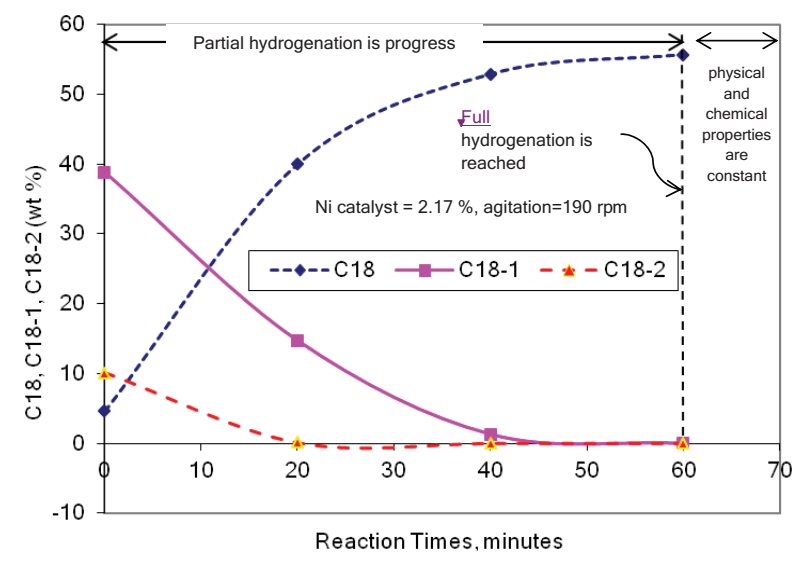

Figure 3. The specific trend's consistency of $\mathrm{C}_{18,} \mathrm{C}_{18-1}$ and $\mathrm{C}_{18-2}$ composition for full hydrogenation of SCPOFA. 
The important of trend's consistency of IV for soybean oil hydrogenation are shown in Figure 4 and Figure 5 (Cizmeci et al.) $)^{2}$. These are different as compared to trend's consistency of IV for SCPOFA hydrogenation.

Cizmeci et al. ${ }^{2}$ also obtained the specific trend's consistency (data aren't shown here) of some physical properties of soybean oil hydrogenation for changing Slip Melting Point (SMP), forming of trans isomer, Solid Fat Content (SFC), selective ratio with two types of catalysts-Nysolsel -222 and SP -10 , under the same specific operating conditions, reactor, and agitator type. Different dosage of the same catalyst gives the specific trend's consistency of soybean hydrogenation. Also different catalyst gives different specific trend's consistency. This is supported by Z. Babaee et al. ${ }^{6}$. The experiences in oleo chemical industry show the same trends.

Experimentation was also conducted with other catalysts: Pd/A and Pd/C (Cizmeci et. al.) $)^{3}$. Based on these, the iodine value of Hydrogenated SoyBean Oil (HSBO), and some physical properties of hydrogenated soybean oil, temperature and pressure of reaction in the reactor can be evaluated at a specific levels - at the specific reaction time of specific trend's consistency of hydrogenation soybean oil - easily. This implies that some specs qualities of HSBO can be able to manipulate for intermediate raw materials for the next purification or products or also as the final products in edible oils industry. Again, these are the manipulation of operating condition and of course, product qualities also. Thus the specific trend' consistency of hydrogenation is important to manipulate the operating conditions and product quality of HFA and HONF.

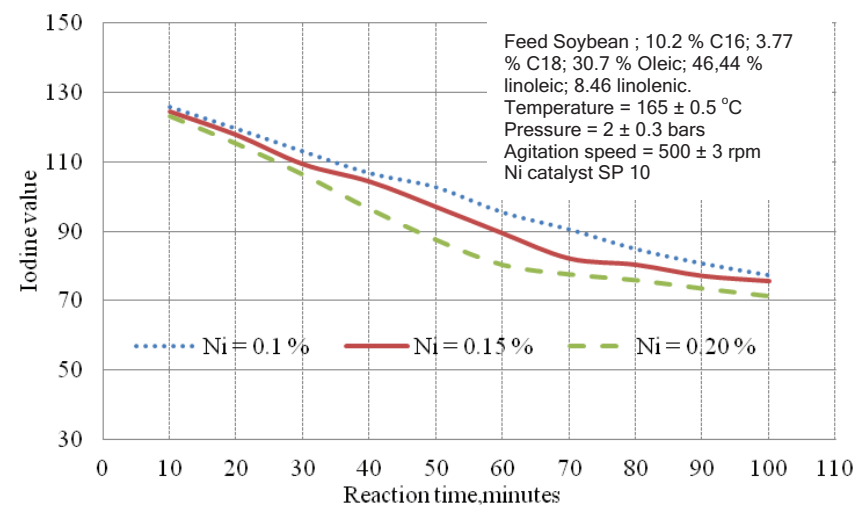

Figure 4. The specific trend's consistency of IV for soybean oil hydrogenation with Nysosel 222.

\subsection{Supported Definitions on Applying Concepts}

Each unit can be used to predict the approximation value of Iodine Value (IV) and Acid Value (AV) of a mixed fatty acid with various compositions.

Acid value of oil and fat can be predicted practically ${ }^{7}$. The same relation can also be used to predict AV of pure fatty acids as a very good approximation. Mixed fatty acid AV can be calculated and predicted too. Based on the unit of fatty acids $\mathrm{AV}$, mixed fatty acid $\mathrm{AV}$ can be predicted from $\mathrm{AV}$ of pure fatty acids and their compositions and also mixed fatty acid IV.

In case of acid value of mixed fatty acids, the experiences indicated that the predicted ones were very close to the real ones of laboratory analysis. These relations are applied in diversification of new products of fatty acid hydrogenation, and distillation process. So, the relations will be able to help to predict and find out the operating conditions, besides the required products quality of HFA and HONF.

\subsection{Creating the Shorter Reaction Time of Hydrogenation}

Fatty acids, oil and fat hydrogenation theory show us, reaction time of partial hydrogenation is shorter than full hydrogenation. The field experiences are also substantiated, proved by the researchers in hydrogenation of soybean oil ${ }^{2,3}$. It is included in the HFA and HONF products to make quality as requested by the costumer or market requirement. It is quite practical to create the suitable and shorter reaction time by using the specific

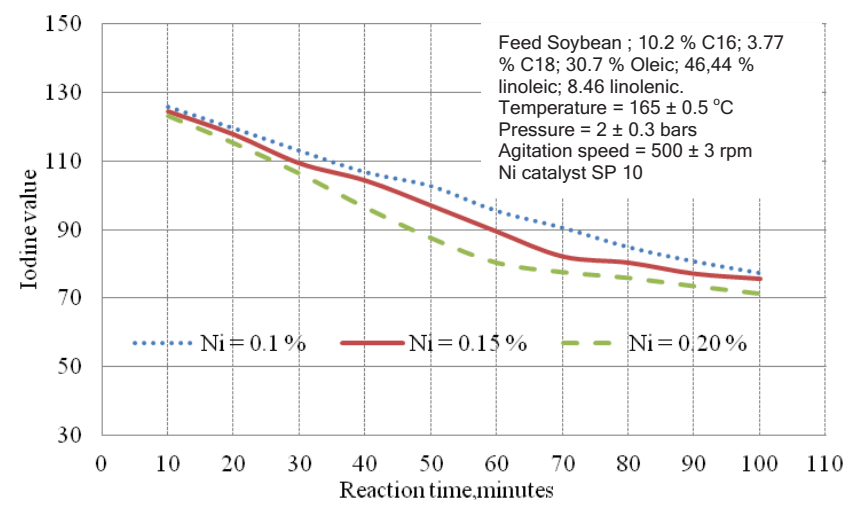

Figure 5. The specific IV trend's consistency of soybean oil hydrogenation with SP 10. 
trend's consistency of Full Hydrogenation (FH). It will not change the beginning parameters of operating conditions reaction, including agitation speed, and catalyst dosage. These concepts will be applied in the field (practical) to provide specific intermediate product of a mixed fatty acid from SCPOFA, an intermediate raw material of mixed fatty acid T40. The operating conditions can be applied directly in the related industries, oleo chemical, and edible oil industry. This intermediate feed is manufactured through degumming, splitting, and $\mathrm{PH}$ processes (Figure 6).

\section{Materials and Methods}

The manufacturing of PHSCPOFA used SCPOFA feed \{have $53 \mathrm{~g} / 100 \mathrm{~g} \mathrm{IV}, 202-205 \mathrm{mg} \mathrm{KOH} / \mathrm{g} \mathrm{AV} \mathrm{min,} \mathrm{and}$ 97.5-98\% SD, GC (\%); $\mathrm{C}_{12}=0.1, \mathrm{C}_{14}=1, \mathrm{C}_{16}=44.3, \mathrm{C}_{18}$ $=5.1, C_{18-1}=38.8, C_{18-2}=9.9, C_{20}=0.3$, Unknown $=0.6$, by trial and error method, based on fatty acids hydrogenation theory ${ }^{8} . \mathrm{H}_{2}$ (g) $99.99 \%$ volume (produced by demin water electroysis), and 30 bars pressure; 25-26 bars reactor pressure; agitation speed of reactor agitator is $190 \mathrm{rpm}$; Ni catalyst Englehard (2.17 kg/ton feed); filter aid $(0.084 \mathrm{~kg} / \mathrm{ton}$ feed); steam medium pressure (12 bars) to heat up SCPOFA to be $145-150^{\circ} \mathrm{C}$, cooling water $\left(26-30^{\circ} \mathrm{C}\right.$ ) to cool down PHSCPOFA to be $85-90^{\circ} \mathrm{C}$, and perform vacuum pressure in reactors and cooling vessels. The scheme of partial hydrogenattion of PHSCPOFA is shown in Figure 7.

\subsection{Experiment Procedures}

\subsubsection{Full Hydrogenation (FH)}

1. Iodine value will be monitored. GC or others properties of feed sample are analysed, depending on the items of quality. For this work IV, and GC are needed to be checked.

2. Full hydrogenation of SCPOFA or intermediate raw material (others FA, oil and fat), depending on the feed are used. This work used SCPOFA as feed.

3. Reactor samples were taken on every 20 minutes (also on the end of reaction), and checked for IV, GC or others; properties of feed sample were analysed (IV, and GC checked).

4. While taking samples (reactor temperature, and pressure), every point of operating conditions was recorded. The operating conditions variable such as catalyst, filter aid dosage (while loading it), start and end temperature, and also agitator speed of reactor are recorded.

5. The specific trend consistency of full hydrogenation was drawnbetween IV, T, and P versus time $(\mathrm{t})$.

6. The specific trend consistency of full hydrogenation was drawn between IV, and composition of $\mathrm{C}_{18}, \mathrm{C}_{18-1}$, $\mathrm{C}_{18-2}$ versus time $(\mathrm{t})$ or other properties to be monitored, and achieved.

7. The obtained figures were used as a base to predict or find out operating conditions of partial hydrogenation, and PHSCPOFA quality. The final product will also

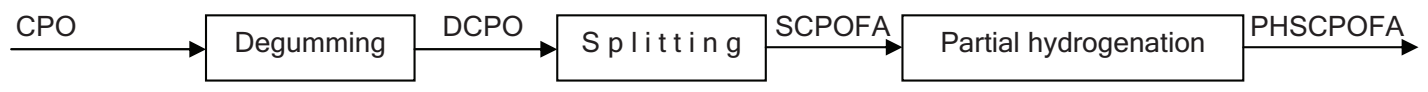

Figure 6. The process steps scheme to manufacture PHCPOFA as an intermedite raw material of mix fatty acid T40. CPO (Crude Palm Oil); DCPO (Degummed CPO); SCPOFA (Splitted CPO Fatty Acid); PHSCPOFA (Partially Hydrogenated SCPOFA) as an intermediate feed of mixed fatty acids T40.

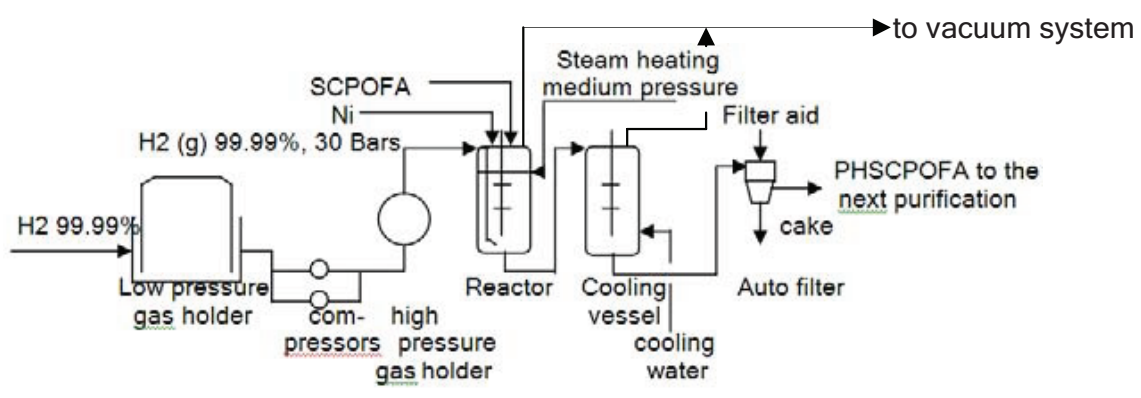

Figure 7. Utilized batch hydrogenation flow diagram to hydrogenate SCPOFA partially to manufacture PHSCPOFA or intermediate raw material of T40, Feld and Hanh Style9. 
be manufactured. In this work, T40 is made as an exmple.

\subsubsection{Prediction of Partial hydrogenation $(\mathrm{PH})$ Operating Conditions}

1. Find the specific specification of final products to be provided (mix unsaturated, and saturated of FA, oil and fat) as the base to modify operating conditions of the specific trend consistency of full hydrogenation to produce the specific specification (IV, GC, AV, SV, titer, SFI, etc).

2. Find the specific specification of partial hydrogenated of FA, oil and fat to be produced such as IV, GC, AV, SV, titer, SFI, etc.

3. Plot the specific specification one by one,needed to be achieved on the specific trend consistency of full hydrogenation as the base to run the partial hydrogenation of the used intermediate raw material. The predicted ones of operating conditions are used for partial hydrogenation of the used intermediate raw material. In this work, SCPOFA is used to provide PHSCPOFA.

4. Find the suitable range to pin point the range of specific specification of products and also operating conditions. These will be flexible, easier, and shorter to run the plant experiment/research.

Partial hydrogenation experiment can be done for 2 batches minimum/experiment or relatively depend on the performance of the obtained results with the same start temperature, catalyst, and filter aid dosage, also agitator of FH.

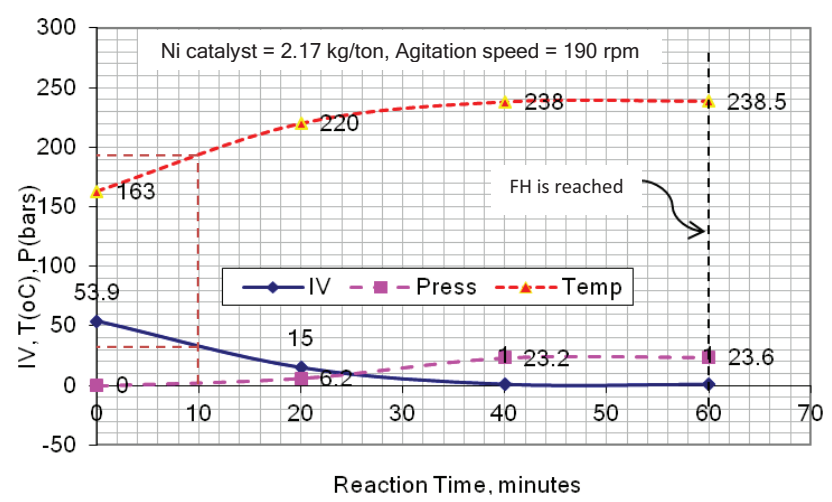

Figure 8. The specific trend's consistency of IV, T and P for hydrogenation of SCPOFA.
The samples are taken, at a level of $500 \mathrm{ml}$ minimum of samples PHSCPOFA, and HSCPOFA, by using the procedure of analysis refering to AOCS 1-62 for FA composition analysis, and Tg 1-64 for FA iodine value analysis, $\mathrm{H} 15-52$ and Te $1 \mathrm{~A}-64$ for acid value analysis ${ }^{10}$.

\section{Results and Discussion}

\subsection{The Specific Trend Consistency of FH}

Th industrial experiment results of the obtained specific trend consistency full hydrogenation of SCPOFA is given in Figure 8 and Figure 9 (represented by 1 st experiment). These figures are the basic to find the quality (IV, AV, and GC), and PH operating conditions of SCPOFA, can be used to predict various of quality, and $\mathrm{PH}$ operating conditions of FA products. Figure 8 shows the method to predict IV, T, dan $\mathrm{P}$ on a reaction time. Time \& the contolled quality parameter are depended on the requirement of precision.

\subsection{Prediction of IV, AV, and GC of PHSCPOFA Product}

The obtained verification of product, and raw material specification (in range) are shown in Table 1. By looking at these range of specifications, the safest point of quality parameters will be designed or predicted.

Mixed fattty acid T40 is a final product which is manufactured through distillation. That 's why it can't be used directly as a base to find out the operating conditions of partial hydrogenation.

In determining the operating conditions, the lowest point of the range of each quality item is used. The

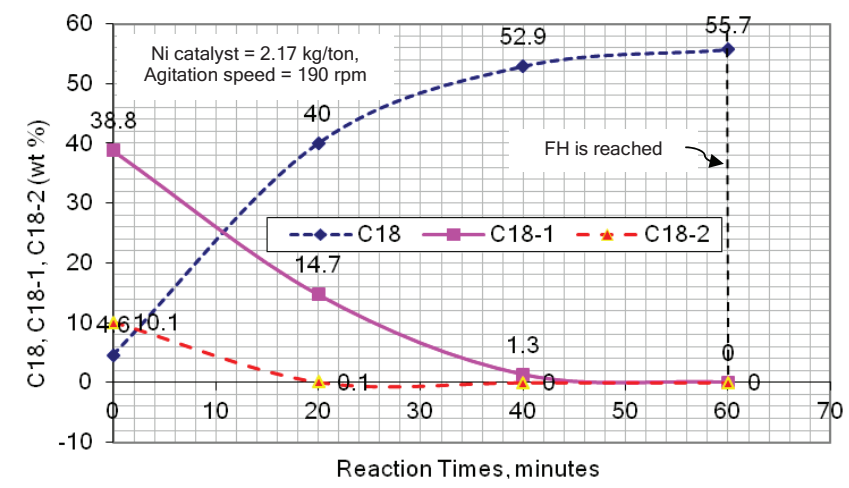

Figure 9. The specific trend's consistency of $\mathrm{C}_{18}, \mathrm{C}_{18-1}$ and $\mathrm{C}_{18-2}$ composition for hydrogenation of SCPOFA. 
prediction of PHSCPOFA quality (as a intermediate raw material T40) and the operating conditions began from these points. The results of the obtained prediction are shown in Table 2.

Predictions began from AV, IV of the end product of T40, started of the lowest point of quality range; \pm 204 for $\mathrm{AV}$, and \pm 35 for IV. The simulated composition is based on the used quality range of AV, and IV of the end product $\mathrm{T}-40$. Simulations are done by considering physical properties of each separated material/Fatty Acid (FA), so it can be done easily in practice.

Hydrolysis of CPO will produce SCPOFA with AV in the range of 202-205 $\mathrm{mg} \mathrm{KOH} / \mathrm{g}$ (This's the key to do this experiment sucessfully). Hydrolysis of CPO is enough to cover AV range of T40 (by keeping $99 \%$ of SD, the conversion), although Partial Hydrogenation will decrease the $\mathrm{AV}$, and IV of PHSCPOFA a little down. The decrease is because of the decrease in major unsaturated $\left(\mathrm{C}_{18-1}, \mathrm{C}_{18-2}\right)$, and increament of saturated $\left(\mathrm{C}_{18}\right)$ fatty acid compositions. For this purpose correct simulation is needed in hydrogenation and distillation.

The results of simulation in Table 2 are made according to the consideration of: $\mathrm{C}_{12}, \mathrm{C}_{14}$, and $\mathrm{C}_{16}$. Compositions are made relatively with CPO or SCPOFA (Table 1), because no hydroegnation at all. The composition of $\mathrm{C}_{18}$ are simulated particularly on $17.6-18.6 \%$. So that the $\mathrm{C}_{18-1}$, and
$\mathrm{C}_{18-2^{\circ}}$. Composition can be first as closer as possible to the T40 composition (partial hydrogenation) - also technical factors are consideredeasier to separate \& decrease $\mathrm{C}_{16}$, and increase $\mathrm{C}_{18}$ in distillation. Because this composition is expected to give the lower boiling point (bp) of FA than that of $\mathrm{FA}$ at $\mathrm{C}_{18}$ range (according to the range of T40). The boiling point of FA increases if saturated FA increases ${ }^{11,12}$. The lower boiling point of FA need the lower heating, and will produce FA with the higher stability ${ }^{11,13}$. The maximum composition of $\mathrm{C}_{20}$, and unk are made according to the long experiences in the oleochemical industry. The composition of $\mathrm{C}_{20}$, and unk can be reduced through distillation. So it will not be a problem in this case. Finaly this is not a problem as long the distillation operating conditions are predicted correctly (not discussed in this artcle).

Purification by distillation changes the PHSCPOFA composition. IV values tend to increase back, because $\mathrm{C}_{12} \& \mathrm{C}_{14}$ are very small, $\mathrm{C}_{16}$ is a part, and $\mathrm{C}_{18}$ is a little bit small, and $\mathrm{C}_{20}$ is very small and separated. So, the increament of $\left(\mathrm{C}_{18-1}\right)$ composition balances until T40 quality can be obtained. Table 3 shows the relative prediction of GC, AV, and IV T40 (that will be produced in distillation) - through the new operating conditions - which is predicted on medium range of T40 quality, until the specifications are obtained. This consideration need enough experiences. Distillation can modify fatty acids produtcs.

Table 1. Final product and raw material specs for partial hydrogenation

\begin{tabular}{|c|c|c|c|c|c|c|c|c|c|c|}
\hline \multirow{2}{*}{ Material } & \multicolumn{8}{|c|}{ Composition (GC), \% by weight } & \multirow{2}{*}{$\begin{array}{l}\mathrm{AV}, \mathrm{mg} \\
\mathrm{KOH} / \mathrm{g}\end{array}$} & \multirow{2}{*}{ IV, $g / 100 \mathrm{~g}$} \\
\hline & $\mathrm{C}_{12}$ & $\mathrm{C}_{14}$ & $\mathrm{C}_{16}$ & $\mathrm{C}_{18}$ & $\mathrm{C}_{18-1}$ & $\mathrm{C}_{18-2}$ & $\mathrm{C}_{20}$ & unkown & & \\
\hline $\mathrm{CPO}$, raw material & 0.1 & 1.0 & 44.3 & 5.1 & 38.8 & 9.9 & 0.3 & 0.6 & 5 & 53 \\
\hline $\mathrm{T}-40$, final product & $1 \max$ & $<4$ & $24-32$ & $24-33$ & $32-42$ & $<2$ & $<2$ & $<0.5$ & $202-207$ & $35-45$ \\
\hline
\end{tabular}

Source: PT. FSC, 2007.

Table 2. The predicted IV, AV \& Composition (GC) of PHSCPOFA

\begin{tabular}{lccccccccccc}
\hline Fatty acid & $\mathrm{C}_{12}$ & $\mathrm{C}_{14}$ & $\mathrm{C}_{16}$ & $\mathrm{C}_{18}$ & $\mathrm{C}_{18-1}$ & $\mathrm{C}_{18-2}$ & $\mathrm{C}_{20}$ & Unk & \multicolumn{3}{c}{ PHSCPOFA } \\
\hline Original IV, g/100 g & - & - & - & - & $89,9^{*}$ & $181^{*}$ & - & - & $\mathrm{IV}, \mathrm{g} / 100 \mathrm{~g}$ & $\mathrm{AV}, \mathrm{mg} \mathrm{KOH} / \mathrm{g}$ \\
Composition, \% w/w & & & & & & & & & & $\mathrm{SD}=99 \%$ & $\mathrm{SD}=98 \%$ \\
$1^{\text {st }}$ & 0.1 & 1,0 & 44.3 & 17.6 & 34,7 & 1,3 & 0.5 & 0.5 & 33,54 & 203.6 & 201.6 \\
$2^{\text {nd }}$ & 0.1 & 1,0 & 44.3 & 18.3 & 33,8 & 1,5 & 0.5 & 0.5 & 33,10 & 203.6 & 201.5 \\
$3^{\text {rd }}$ & 0.1 & 1,0 & 44.3 & 18.2 & 33,9 & 1,5 & 0.5 & 0.5 & 33,18 & 203.6 & 201.5 \\
$4^{\text {th }}$ & 0.1 & 1,0 & 44.3 & 18.6 & 33,6 & 1,4 & 0.5 & 0.5 & 32,73 & 203.6 & 201.5 \\
\hline
\end{tabular}

Notes: *Unichema International, 1998. 
Therefore, that are so normal that the medium point of final product specific ranges are made as a basic to predict PHSCPOFA quality and of course also the operating conditions. The medium point of the quality is the safest point in this prediction.

The lower fatty acid $\mathrm{C}_{12}$ and $\mathrm{C}_{14}$ is separated in very small quantity, because it amount's very small in the feed, so it's predicted almost same after distillation. This is just to help to improve T40 colour, its composition doesn't change much. This composition help much to obtaine the higher AV of final product (T40), and its quality specification. This composition is so small, and very difficult to reduce through distillation or fractionation as the experiences show us this facts. This fact supported us stronger to keep it in the prediction. In the IV, prediction reuires only unsaturated $\mathrm{C}_{18-1}$, and $\mathrm{C}_{18-2}$, In this case, the composition of both FA is the key to find the values of IV total suitably the new operating conditions (not discussed here). The equation for prediction of the total values of AV, IV of mixed FA are

Total AV $=\sum$ (weight percent of each FA $\times$ AV of each FA)

Total IV $=\sum$ (weight percent of each unsaturated FA $\times$ IV of each unsaturated FA)

Both tables above are the fundamental to predict the operating conditions of partial hydrogenation, and can be rearranged with the further experiment results, based on the final product quality that will be manufactured.

\subsection{Proving of the IV, and GC Prediction by using the Specific Trend's Consistency of FH}

The prediction of PHSCPOFA IV is $33-34 \mathrm{~g} / 100 \mathrm{~g}$ in PH of SCPOFA that will be produced. It's based on the predicted quality of PHSCPOFA (Table 2 ). The hydrogenation results, which are recorded on 1st 20' are shown in Table 4 and Table 5 (Ni catalyst, $2.17 \mathrm{kgs} /$ ton; agitation speed, $190 \mathrm{rpm})$, and are obtained IV as (14.7-15.0)< 33-34 g/100 g, These are not same. The supposed reaction time $(\mathrm{t})$ has, rherefore, to be under 20. For the excelent decision on the correct reaction time for $\mathrm{PH}$, it is very well used for the specific trend's consistency of the obtained FH (1st experiment as a representative figure) in the Figure 10.

The changes of IV, operating conditions, and PHSCPOFA composition which are obtained in $1^{\text {st }}$ experiment is depicted in Figure 10, Figure 11 and $2^{\text {nd }}$ experiment in Table 6. The changes of composition of the same FA for

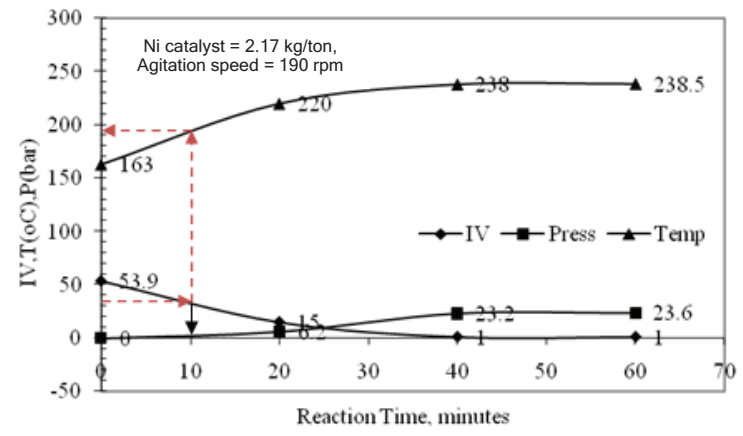

Figure 10. The specific trend's consistency of IV, and operating conditions ( $1^{\text {st }}$ experiment) of PSCPOFA.

Table 3. The predicted composition (GC) \& AV, and IV of T -40 to be manufactured ${ }^{12}$

\begin{tabular}{|c|c|c|c|c|c|c|c|c|c|}
\hline Fatty acid & $\mathrm{C}_{12}$ & $\mathrm{C}_{14}$ & $\mathrm{C}_{16}$ & $\mathrm{C}_{18}$ & $\mathrm{C}_{18-1}$ & $\mathrm{C}_{18-2}$ & $\mathrm{C}_{20}$ & \multirow{2}{*}{$\begin{array}{c}\text { Mixed } \\
\text { AV }\end{array}$} & \multirow{2}{*}{$\begin{array}{c}\text { Mixed } \\
\text { IV }\end{array}$} \\
\hline Original AV, mg KOH/g & $280.1^{*}$ & $245^{*}$ & $216^{*}$ & $197.2^{*}$ & $199^{*}$ & $200^{*}$ & $179^{*}$ & & \\
\hline Predicted Composition, \% & 0.1 & 1.0 & 28.0 & 33.0 & 36.0 & 1.5 & 0.4 & 204.1 & 35.0 \\
\hline
\end{tabular}

Table 4. IV Reduction and the operating conditions of PHSCPOFA ( $1^{\text {st }}$ experiment)

\begin{tabular}{cccc}
\hline $\begin{array}{c}\text { Reaction } \\
\text { time }(\mathrm{t})\end{array}$ & IV, g/100 g & $\begin{array}{l}\text { Pressure, } \\
\text { bars }\end{array}$ & Temperature, ${ }^{\circ} \mathrm{C}$ \\
\hline $0^{\prime}$ & 53.9 & 0.0 & 163.0 \\
$20^{\prime}$ & 15.0 & 6.2 & 220.0 \\
\hline
\end{tabular}

Table 5. IV Reduction, and operating conditions of PHSCPOFA ( $2^{\text {nd }}$ experiment)

\begin{tabular}{cccc}
\hline $\begin{array}{c}\text { Reaction } \\
\text { time }(\mathrm{t})\end{array}$ & IV, g/100 g & $\begin{array}{l}\text { Pressure, } \\
\text { bars }\end{array}$ & Temperature, ${ }^{\circ} \mathrm{C}$ \\
\hline $0^{\prime}$ & 53.7 & 0.0 & 163.0 \\
$20^{\prime}$ & 14.7 & 6.4 & 215.0 \\
\hline
\end{tabular}


$2^{\text {nd }}$ experiment which is shown in in Table 6, isn't the same as the obtained prediction in Table 2 above. The value of IV and operating conditions can predict specific trend's consistency of FH SCPOFA (represented by $1^{\text {st }}$ experiment) on 10' minutes reaction time, and is shown in Figure 10.

Then, the results of prediction are tried in $3^{\text {rd }}$ experiment, and the results are shown in Table 7 (just show untill 10 ' reaction time), and the obtained IV of PHSCPOFA which is in line with the prediction in Table 2, are obtained as well. The over all results of IV changes, hydrogenation operating conditions, and PHSCPOFA composition as shown in Figure 11 and Figure 12.

Table 7 shows that IV results are a little bit lower than the results of prediction in Table 2. They are different a little bit, and can be used as distillation feed to manufacture T40. If IV will be increased, $\mathrm{PH}$ of reaction time can be decreased to lower than 10 , so that $\mathrm{C}_{18-1}$ can be lower or $\mathrm{C} 18$ can be higher, so it can reduce the load of distillation to increase up $\mathrm{C}_{18-1}$ partcularly, and distillation yield be higher. The same method can be used on the specific trend's consistency ( $3^{\text {rd }}$ experiment) which are shown in Figure 11 and Figure 12- to be predicted at a little bit $<$ 10 'reaction time.

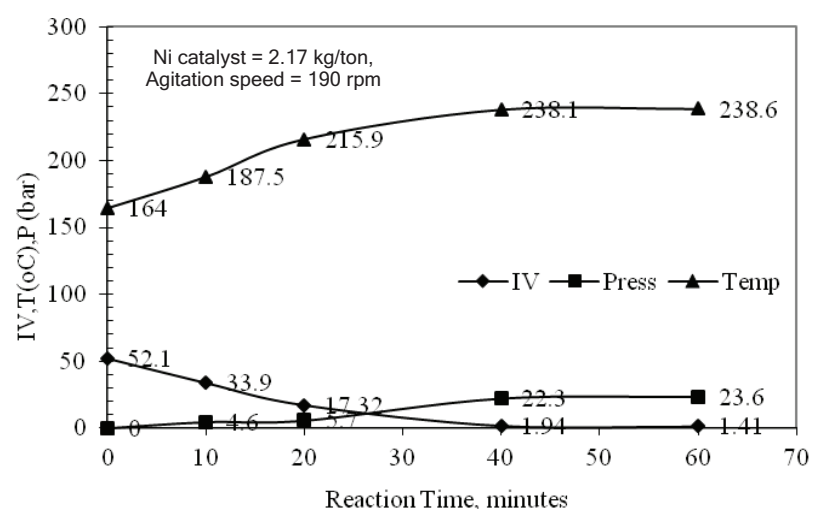

Figure 11. The specific trend's consistency of IV, and operating conditions of PHSCPOFA ( $3^{\text {rd }}$ experiment).

\section{Conclusion and Suggestions}

The specific trend's consistency of FH of SCPOFA is usefull to manufacture PHSCPOFA (intermediate raw material of T40). We suggest its practical usability for quality operating conditions for manufacturing. PHSCPOFA. The obtained operating conditions in this experiment are: Initial temperature To $=164^{\circ} \mathrm{C}$; Final temperature $\mathrm{Ti}=187.5^{\circ} \mathrm{C}$; Reactor pressure $\mathrm{P}=4.6$ bars; Reaction time $\mathrm{t}=10$ minutes, Ni catalyst dosage $=2.17$ $\mathrm{kg} / \mathrm{ton}$; and Agitation speed $=190 \mathrm{rpm}$.

The specific trend's consistency of FH are important, and can be applied practically to predict quality and FA $\mathrm{PH}$ operating conditions This method is strongly advocated for use in $\mathrm{PH}$ of oils and fats in edible industry. The principle of FA, and oils and fats hydrogenation are the same.

It's recomended strongly to get samples more frequently - in the time interval of $<10$ ' reaction time (Ex: every 5 minutes) to analysis IV, and GC also and recording well the related main operating conditions of $\mathrm{FH}$ of SCPOFA. The most precise specific trend's consistency of FH of SCPOFA can be drawn, so the simulation and prediction can be done precisely.

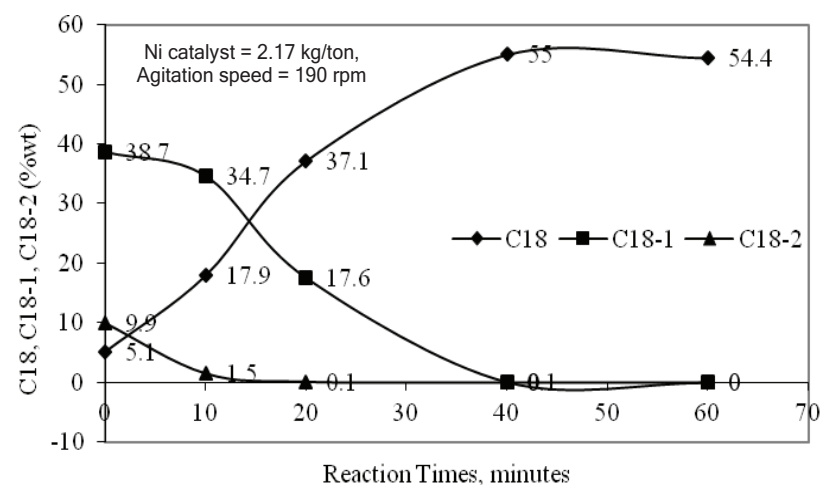

Figure 12. The specific trend's consistency of $\left(\mathrm{C}_{18}, \mathrm{C}_{18-1}\right.$ and $\mathrm{C}_{18-2}$ ) composition of PHSCPOFA ( ${ }^{\text {rd }}$ experiment).

Table 6. The obtained composition of PHSCPOFA for 20 minutes reaction time ( $2^{\text {nd }}$ experiment)

\begin{tabular}{lcccccccc}
\hline Fatty acid & $<\mathrm{C}_{14}$ & $\mathrm{C}_{16}$ & $\mathrm{C}_{18}$ & $\mathrm{C}_{18-1}$ & $\mathrm{C}_{18-2}$ & $\mathrm{C}_{20}$ & unk & $\mathrm{IV}$ \\
\hline Feed (reaction time, 0') & 1.0 & 44.6 & 4.6 & 38.8 & 10.1 & 0.3 & 0.5 & 53.7 \\
Reaction time, 20' & 1.0 & 43.6 & 40 & 14.7 & 0.1 & 0.5 & 0.1 & 14.7 \\
\hline
\end{tabular}


Table 7. The third experiment: IV reduction, and operating conditions of PHSCPOFA

\begin{tabular}{lccc}
\hline $\begin{array}{l}\text { Reaction time } \\
(\mathrm{Rt})\end{array}$ & $\mathrm{IV}, \mathrm{g} / \mathbf{1 0 0} \mathrm{g}$ & $\begin{array}{l}\text { Pressure, } \\
\text { bars }\end{array}$ & Temperature, ${ }^{\circ} \mathrm{C}$ \\
\hline $1^{\text {st }}$ experiment & & & \\
$10^{\prime}$ & 33.90 & 4.6 & 187.5 \\
$2^{\text {nd }}$ exeriment & & & \\
$10^{\prime}$ & 32.73 & 5.0 & 188.7 \\
$3^{\text {rd }}$ experiment & & & \\
$10^{\prime}$ & 32.21 & 4.9 & 187.6 \\
\hline
\end{tabular}

Notes: The start temperature for the experiments; $1^{\text {st }}\left(164^{\circ} \mathrm{C}\right)$, $2^{\text {nd }}\left(162.8^{\circ} \mathrm{C}\right)$, and $3^{\text {rd }}\left(161.2^{\circ} \mathrm{C}\right)$.

\section{Acknowledgement}

Thanks to PT. Flora Sawita Chemindo management that gave the author facilities to conduct this work (product diversification of PHSCPOFA as an intermediate raw material of final product of T40) entirely in their plant premises.

\section{References}

1. Patterson HBW. Hydrogenation of fats and oils: theory and practice. United State of America: AOCS Press; 2009.
2. Cicmezi M, Musavi A, Kayahan M, Tekin A. Monitoring of hydrogenation with various catalyst ratios. J Am Oil Chem Soc. 2005; 82(12):926-27.

3. Cicmezi M, Musavi A, Kayahan M, Tekin A. Comparision of two palladium catalysts on different supports during hydrogenation. J Am Oil Chem Soc. 2006; 83(12): 1063-68.

4. Hui YH. Bailey's industrial oil and fat products. 5th ed. New York: John Wiley \& Sons; 1996.

5. Swern D. Bailey's industrial oil and fat products. 3rd ed. New York: John Wiley \& Sons; 1964.

6. Babaee Z, Nikoopour H, Safafar H. A comparation of commercial nickel catalyst effects on hydrogenation soy bean oil. World Appl Sci J. 2007; 2(6):621-26.

7. Edgar W. The manufacture of soaps, others detergents and glycerine. England: Ellis Horwood Limited; 1985.

8. Shahidi F. Bailey's industrial oil and fat products. 6th ed. Hoboken, N J : John Wiley \& Sons; 2005.

9. PT. FSC fatty acid production report, medan, Indonesia; 2005.

10. David F. Official method and recommended practices of the AOCS. 5th ed. USA: AOCS Press; 2006.

11. Ketaren S. Pengantar teknologi minyak dan lemak pangan. Jakarta: UI Press; 2009

12. Unichema International. Fatty acid data book. 2nd ed. Federal Republic of German; 1998.

13. Muhammad YR. Optimization of palmitic acid composisiton in crude oleic acid to provide specifications of titer and cloud point of distillate oleic acid using a flash distiller distillate oleic acid using a flash distiller. J Technol \& Sci IPTEK. 2010; 21(4):203-13. 